\title{
ARTICLE Downregulated AKT-mTOR signaling pathway proteins in dorsolateral prefrontal cortex in Schizophrenia
}

\author{
Radhika Chadha iD ${ }^{1}$ and James H. Meador-Woodruff ${ }^{1}$
}

\begin{abstract}
Abnormal neurotransmission is central to schizophrenia (SZ). Alterations across multiple neurotransmitter systems in SZ suggest that this illness may be associated with dysregulation of core intracellular processes such as signaling pathways that underlie the regulation and integration of these systems. The AKT-mTOR signaling cascade has been implicated in SZ by gene association, postmortem brain and animal studies. AKT and mTOR are serine/threonine kinases which play important roles in cell growth, proliferation, survival, and differentiation. Both AKT and mTOR require phosphorylation at specific sites for their complete activation. mTOR forms two functionally distinct multiprotein complexes, mTOR Complex 1 (mTORC1) and Complex 2 (mTORC2). mTORC1 mediates ribosome biogenesis, protein translation, and autophagy, whereas mTORC2 contributes to actin dynamics. Altered protein synthesis and actin dynamics can lead to an abnormal neuronal morphology resulting in deficits in learning and memory. Currently, there is a lack of direct evidence to support the hypothesis of disrupted mTOR signaling in SZ, and we have addressed this by characterizing this signaling pathway in SZ brain. We found a reduction in AKT and mTOR protein expression and/ or phosphorylation state in dorsolateral prefrontal cortex (DLPFC) from 22 pairs of SZ and matched comparison subjects. We also found reduced protein expression of $G \beta L$, a subunit protein common to both mTOR complexes. We further investigated mTOR complex-specific subunit composition and phosphorylation state, and found abnormal mTOR expression in both complexes in SZ DLPFC. These findings provide evidence that proteins associated with the AKT-mTOR signaling cascade are downregulated in SZ DLPFC.
\end{abstract}

Neuropsychopharmacology (2020) 45:1059-1067; https://doi.org/10.1038/s41386-020-0614-2

\section{INTRODUCTION}

Abnormal neurotransmission is a key feature of schizophrenia (SZ) [1]. In SZ brain, myriad changes across multiple neurotransmitter systems (dopamine, serotonin, GABA, glutamate, and others) [2-7] suggest that rather than being a disorder of one specific neurotransmitter pathway, SZ may instead be a disturbance of core intracellular processes that regulate multiple systems. Dysregulation of intracellular signaling pathways, especially pathways that integrate signals from multiple neurotransmitter systems, have been implicated in the pathophysiology of this disorder. For example, there are abnormalities in SZ associated with the mitogen activated protein kinase (MAPK), 3'-5' cyclic adenosine monophosphate (CAMP), and glycogen synthase kinase-3 beta (GSK3 $\beta$ ) signaling pathways [8-11]. The AKT-mTOR signaling pathway is another intracellular cascade that modulates important cellular events, including protein synthesis and actin dynamics, both of which are critical for neurotransmission and have been implicated in the pathophysiology of SZ [12]. This pathway plays a pivotal role in synaptic plasticity and facilitates long-term memory formation [13, 14]. The mechanisms underlying synaptic strength, especially long-term potentiation and long-term depression, depend on new protein synthesis and cytoskeletal rearrangement, key functions of mTOR signaling. Although the AKT-mTOR pathway is associated with intracellular processes that are disrupted in SZ, it has not yet been well characterized in this illness.
The AKT-mTOR signaling pathway is tightly regulated by differential phosphorylation of key proteins (Fig. 1). AKT is a serine/threonine (Ser/Thr) kinase that modulates signals from growth factors, neurotransmitters, and cytokines, and regulates cell growth, proliferation, survival, differentiation, and metabolism. It requires phosphorylation at two key residues for full activation, Ser 473 (S473) and Thr 308 (T308) [15]. mTOR is also a Ser/Thr kinase which is central to two structurally and functionally distinct complexes, mTOR complex I (mTORC1) and mTOR complex II (mTORC2). AKT is functionally interconnected with both mTOR complexes. Upstream of mTORC1, AKT inhibits the association of tuberous sclerosis complexes I and II (TSC1 and TSC2). TSC1/TSC2 heterodimers activate Ras homolog enriched in brain (Rheb), which in turn activates mTORC1 [12]. mTORC2 phosphorylates AKT at S473, while phosphoinositide-dependent kinase 1 (PDK1) phosphorylates AKT at T308 [16]. Like AKT, mTOR also requires phosphorylation for full activation in both complexes at two residues, Ser 2448 (S2448) and Ser 2481 (S2481), which are regulated by distinct kinases [17]. mTORC1 is a multiprotein assembly that includes regulatory associated protein of mTOR (Raptor), Deptor, proline-rich AKT substrate $40 \mathrm{kDa}$ (PRAS40), G protein beta subunit like (GßL or $\mathrm{mLST}$ ) and mTOR itself [18]. This complex facilitates ribosome biogenesis by phosphorylating p70 S6 kinase (p70 S6K) [19], mediates protein translation by phosphorylating eukaryotic translation initiation factor $4 \mathrm{E}$-binding protein, and inhibits autophagy by phosphorylating ULK1 [20].

\footnotetext{
${ }^{1}$ Department of Psychiatry and Behavioral Neurobiology, University of Alabama at Birmingham, 1719 6th Avenue South, Birmingham, AL 35294-0021, USA
} Correspondence: Radhika Chadha (radhikac@uab.edu)

Received: 11 October 2019 Revised: 7 January 2020 Accepted: 9 January 2020

Published online: 17 January 2020 
Cell growth, survival, proliferation, metabolism

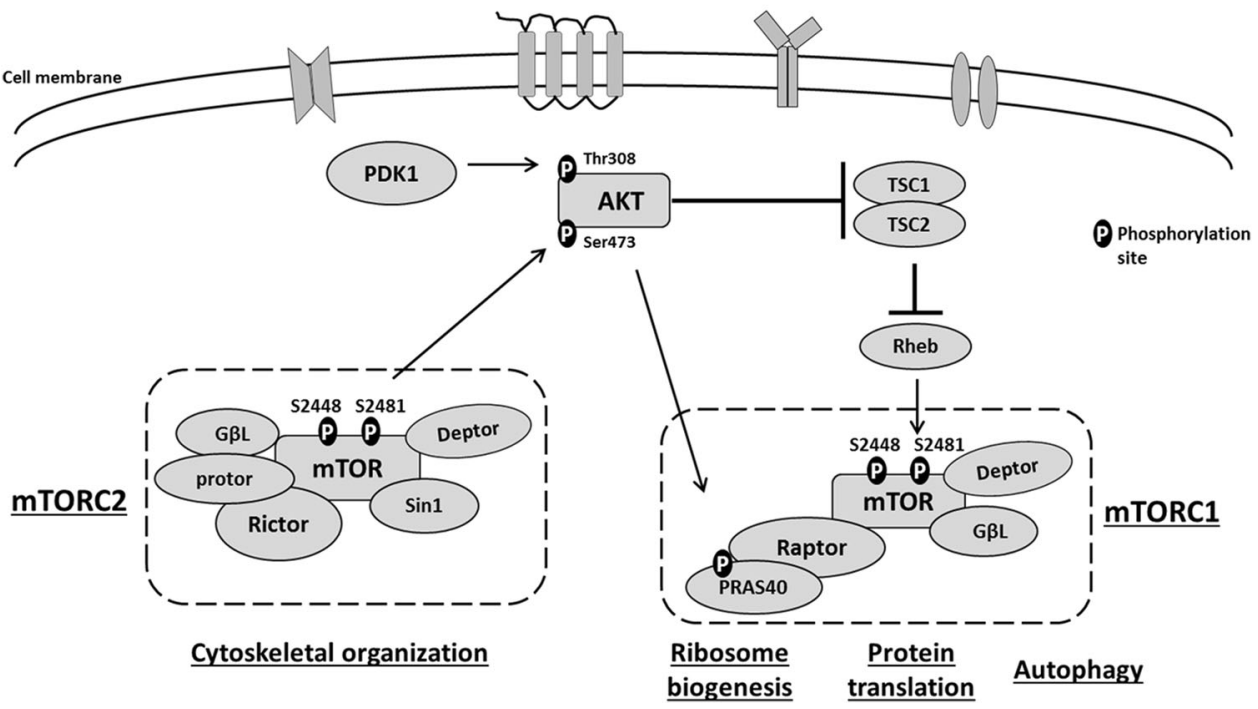

Fig. 1 AKT-mTOR signaling pathway. AKT and mTOR are key kinases in this signaling cascade. They both require phosphorylation for complete activation. mTOR forms two distinct complexes: mTORC1 and mTORC2. mTORC1 comprises of mTOR, Raptor, Deptor, PRAS40, and $\mathrm{G} \beta \mathrm{L}$, and facilitates ribosome biogenesis, protein translation and autophagy. mTORC2 consists of mTOR, Rictor, Deptor, Protor, G $\beta \mathrm{L}$, and Sin 1 , and modulates cytoskeleton organization. AKT is functionally interconnected with mTOR complexes since it positively regulates mTORC1 activity via Rheb, whereas mTORC2 positively regulates AKT activity by phosphorylating it at S473.

Raptor, one of the constituent proteins of mTORC1, is rapamycinsensitive and crucial for the kinase activity of this complex [17]. Deptor and PRAS40 inhibit mTORC1 activity [20, 21], while GßL enhances the kinase activity of the complex [22]. Like mTORC1, mTORC2 is a multiprotein assembly that includes rapamycin insensitive companion of mTOR (Rictor), Deptor, Protor, G $\beta$ L, stress activated protein kinase-interacting (Sin1), and mTOR itself. Rictor recruits mTORC2 substrates, while Protor and Sin 1 promote RictormTOR binding to stabilize the complex $[22,23]$. A key function of mTORC2 is modulation of actin dynamics by regulating the phosphorylation of protein kinase $C a[18,20]$.

AKT-mTOR signaling regulates protein synthesis and actin cytoskeleton, which are critical events for long-term memory formation as well as neural plasticity [24]. Although this signaling pathway has not been well characterized in SZ, the intracellular processes of actin polymerization, dendritic spine density, and protein synthesis have all been reported to be disrupted in multiple brain regions in SZ patients [25-28]. AKT mRNA, protein, and activity have been found to be reduced in postmortem frontal cortex and blood isolated from SZ subjects [29, 30]. Pharmacological inhibition of mTORC1 in mice blocks long-term synaptic plasticity and memory storage by inhibiting protein synthesis [31]. Dendritic spine alterations, which are associated with abnormal actin dynamics, have been identified in multiple brain regions in SZ [27]. Protein synthesis has been found to be reduced in SZ patient-derived olfactory neurospheres (ONS). Data integration analysis of proteomic and transcriptomic datasets from ONS cells, as well as genome-wide association data from SZ patients, highlight that dysregulated mTOR signaling is associated with this illness [25]. These findings reflect a growing body of evidence that suggests alterations in the expression and activity in the AKT-mTOR signaling pathway in SZ. Therefore, we hypothesized that abnormalities in the AKT-mTOR signaling pathway are associated with SZ pathophysiology. To test this, we measured the expression of key proteins and their phosphorylation state in this signaling pathway in dorsolateral prefrontal cortex (DLPFC) from SZ and comparison subjects. In addition, we isolated mTOR complexes and assessed complex-specific abnormalities in subunit composition and protein phosphorylation in these subjects.
Table 1. Summary of subject demographics.

\begin{tabular}{lll}
\hline & Comparison & Schizophrenia \\
\hline$N$ & 22 & 22 \\
Sex & $5 \mathrm{~F} / 17 \mathrm{M}$ & $5 \mathrm{~F} / 17 \mathrm{M}$ \\
Age (years) & $70.5 \pm 14.5$ & $69.6 \pm 11.6$ \\
Tissue $\mathrm{pH}$ & $6.5 \pm 0.3$ & $6.4 \pm 0.2$ \\
$\mathrm{PMI}(\mathrm{h})$ & $13.4 \pm 7$ & $14.8 \pm 5.3$ \\
On/Off/Unknown Rx & $0 / 22$ & $13 / 7 / 2$ \\
Race & $13 \mathrm{~W} / 3 \mathrm{~B} / 1 \mathrm{~A} / 5 \mathrm{H}$ & $17 \mathrm{~W} / 3 \mathrm{~B} / 0 \mathrm{~A} / 2 \mathrm{H}$ \\
Smoking status & $3 \mathrm{Y} / 10 \mathrm{~N} / 5 \mathrm{E} / 4 \mathrm{U}$ & $9 \mathrm{Y} / 5 \mathrm{~N} / 3 \mathrm{E} / 5 \mathrm{U}$ \\
\hline
\end{tabular}

Values presented are means \pm standard deviation

Sex: $F$ female, $M$ male; $P M I$ postmortem interval; $R x$ : On treated with antipsychotic medications at time of death; Off off antipsychotic medication for $>6$ weeks prior to death; Race: $W$ white, $B$ black, $A$ Asian, $H$ hispanic; Smoking: $N$ never, $Y$ yes, $E$ no smoking at least 6 months, $U$ unknown

\section{METHODS}

\section{Subjects}

Postmortem human brain samples were provided through the $\mathrm{NIH}$ Neurobiobank. All samples were from the Mount Sinai/Bronx Veterans Administration Medical Center brain collection. Assessment, consent, and postmortem procedures were conducted as required by the Institutional Review Boards of Pilgrim Psychiatric Center, Mount Sinai School of Medicine, and the Bronx Veterans Administration Medical Center as has been previously described $[32,33]$. Twenty-two subjects diagnosed with SZ using DSM-III-R criteria were pairwise matched with comparison subjects by sex, age, tissue $\mathrm{pH}$, and postmortem interval (PMI) (Table 1). The majority of SZ subjects utilized in this study were treated with typical (first generation) antipsychotics. Nonpsychiatrically ill subjects were selected from this brain bank to serve as a comparison group. Criteria for subject exclusion included a history of substance abuse, death by suicide, or coma for $>6 \mathrm{~h}$ prior to death. Subjects had no evidence of neuropathology, or signs of 
neurodegenerative disorders such as Alzheimer's disease at assessment.

\section{Tissue preparation}

Whole brains were dissected into $10 \mathrm{~mm}$ slabs in the coronal plane. Gray matter from DLPFC (Broadmann areas 9/46) was dissected into $1 \mathrm{~cm}$ cubes from SZ and comparison subjects and stored at $-80^{\circ} \mathrm{C}$ until use. Samples were homogenized using a Power Gen 125 homogenizer (Thermo Fisher Scientific, Rockford, Illinois) in Sucrose-Tris buffer (0.32 M Sucrose, $5 \mathrm{mM}$ Tris- $\mathrm{HCl}$ ph 7.4) containing protease and phosphatase inhibitor tablets (Complete Mini, EDTA-free and PhosSTOP, both from Roche Diagnostics, Mannheim, Germany). Homogenates were sonicated on ice for $5 \mathrm{~s}$ at setting 3 using a Sonic Dismembrator (Fisher Scientific, Pittsburgh, Philadelphia) for western blot experiments. Protein concentration was determined using a BCA assay kit (Thermo Fisher Scientific, Rockford, Illinois) and homogenates were aliquoted and stored at $-80^{\circ} \mathrm{C}$ until use.

Rodent antipsychotic drug treatment

We modeled chronic antipsychotic treatment utilizing male Sprague-Dawley rats $(250 \mathrm{~g})$. They were housed in pairs for the duration of the study ( 9 months), during which they were treated with either haloperidol decanoate $(28.5 \mathrm{mg} / \mathrm{kg}, n=10)$ or vehicle (sesame oil, $n=10$ ) every 3 weeks for a total of 12 intramuscular injections, as published previously [28, 32-34]. The dose of haloperidol, duration of treatment, and the drug delivery protocol was chosen based on previously published studies describing antipsychotic treatment in rats $[35,36]$. The animals were sacrificed by decapitation and the brains immediately harvested. The frontal cortex was dissected on ice, snap frozen on dry ice, and stored at $-80^{\circ} \mathrm{C}$. Samples were homogenized in Sucrose-Tris buffer $(0.32 \mathrm{M}$ Sucrose, $5 \mathrm{mM}$ Tris- $\mathrm{HCl}$ pH 7.4) containing protease and phosphatase inhibitor tablets (Complete Mini, EDTA-free and PhosSTOP, both from Roche Diagnostics, Mannheim, Germany). The protocol was approved by the Institutional Animal Care and Use Committee of the University of Alabama at Birmingham.

\section{Co-immunoprecipitation}

To investigate abnormalities in subunit composition and protein phosphorylation in mTOR complexes, we optimized coimmunoprecipitation of mTOR complexes using proteins specific for each complex, Raptor for mTORC1 and Rictor for mTORC2. To our knowledge, there are no existing reports of using this protocol in postmortem human brain. Our lab has previously shown immunoisolation of early endosomes with early endosome antigen-1 (EEA1) protein [37]. We utilized a similar protocol and optimized it for mTOR complexes. A $100 \mu \mathrm{g}$ aliquot of each homogenized sample in Sucrose-Tris buffer (not sonicated) was used for all co-immunoprecipitations with $100 \mu \mathrm{l} \mathrm{M-280} \mathrm{magnetic}$ sheep anti-rabbit IgG Dynabeads (Life Technologies, Grant Island, New York). $10 \mu \mathrm{g}$ anti-raptor (Millipore 05-1470) or anti-rictor (Abcam 104838) antibodies were used for co-immunoprecipitation, determined after optimizing the protocol to obtain maximum depletion from the supernatant (Fig. 4 and Supplementary Fig. S2). Cold TBS (Tris-buffered saline) with $0.1 \%$ Tween- 20 was used to wash beads before and after incubations. Magnetic beads were washed three times and incubated with antibody at $4{ }^{\circ} \mathrm{C}$ with rotation for $1 \mathrm{~h}$. For Rictor co-immunoprecipitation, beads were blocked for $15 \mathrm{~min}$ at $4{ }^{\circ} \mathrm{C}$ with rotation using $5 \%$ BSA (Bovine serum albumin) in TBS, before incubating with antibody. Excess antibody was removed by washing three times and the antibodybound beads were incubated with homogenate at $4{ }^{\circ} \mathrm{C}$ with rotation for $2 \mathrm{~h}$. Supernatant was collected and beads were washed before elution with $2 \mathrm{X}$ reducing buffer for $10 \mathrm{~min}$ at $70^{\circ} \mathrm{C}$. Western blot analysis was used to confirm the immunoprecipitation of mTOR complexes.
Western blot analysis

Western blot analyses were performed as previously described $[32,38-40]$. Samples were boiled in $6 \mathrm{X}$ reducing buffer $(\mathrm{pH} 6.8)$ to a final $1 \mathrm{X}$ buffer concentration at $70^{\circ} \mathrm{C}$ for $10 \mathrm{~min}$. Fifteen micrograms of protein was loaded in each lane of a $4-12 \%$ gradient polyacrylamide bis-tris gel or 3-8\% gradient polyacrylamide tris-acetate gel (Invitrogen, Carlsbad, California). Samples were run in duplicate for $1.5 \mathrm{~h}$ at $150 \mathrm{~V}$. Proteins were transferred to nitrocellulose membranes using trans-blot SD semi-dry transfer (Bio-rad, Hercules, California) at $16 \mathrm{~V}$ for $36 \mathrm{~min}$. Membranes were blocked and probed using conditions optimized for each antibody (Supplementary Table S1) such that detection was within the linear range of the assay and each primary antibody was present in excess. TBS with $0.1 \%$ Tween-20 was used for rinsing the membranes. No significant difference in VCP expression was observed between SZ and comparison groups, consistent with previous studies [34, 41, 42]. Total expression of proteins was used to normalize the expression of their phosphorylated form. For western blot of mTOR complexes, half of each co-immunoprecipitated product was loaded in each lane, and the protein expression of the immunoprecipitating protein (Raptor/Rictor) was utilized to normalize the expression of target protein as an intra-lane loading control. Membranes were imaged using a LiCor Odyssey scanner (LiCor, Lincoln, NE).

\section{Data analysis}

Image Studio Lite version 5.2 (LiCor) was used to measure the signal intensity of each protein. Boxes were manually placed around each band at the expected molecular weight of each protein and background was subtracted. Data from duplicate lanes were averaged for analysis. To determine whether data were normally distributed, D'Agostino and Pearson omnibus normality test was performed for each dependent variable. Outliers were detected by the ROUT method $(Q=1 \%)$ and removed. Normally distributed data from the subjects were analyzed using paired Student's $t$ tests and data that were not normally distributed were analyzed using Wilcoxon matched-pairs signed rank tests. Pearson correlation coefficients were utilized to determine the association between dependent measures and age, $\mathrm{PMI}$, and tissue $\mathrm{pH}$ values. Proteins measured in haloperidol-treated rats were analyzed using unpaired $t$ tests or Mann-Whitney $U$ tests. For all analyses, $a=$ 0.05. All data analyses were performed using GraphPad Prism version 7.00 for Windows (GraphPad Software, La Jolla, California).

In silico analysis

We evaluated patterns of differential transcript/protein expression associated with the AKT-mTOR signaling pathway in SZ using Kaleidoscope, an interactive web application developed by Robert E. McCullumsmith's lab at the University of Toledo (https://kalganem. shinyapps.io/BrainDatabases/). This application utilizes publicly available databases and is connected to bioinformatics tools like Brain RNAseq, Brain Cloud, STRING, iLINCS, and the Allen Brain Atlas. We selected datasets in the application to determine cell-specific differential expression in past SZ studies. These datasets included blood mRNA microarray expression obtained from SZ and control subjects [43], data generated from laser capture microdissected pyramidal neurons from superficial (lamina II-III; Super) or deep (lamina V-Vl; Deep) cortical layers of DLPFC (unpublished data), RNA sequencing and proteomic data from neurons derived from induced pluripotent stem cells (iPSCs) from SZ patients with a DISC1 mutation and control subjects [44], RNA sequencing data from neurons and neuron progenitor cells derived from iPSCs from early onset SZ patients and controls [45], and microarray expression of pyramidal cells in DLPFC layers 3 or $5(L 3 / L 5)[46,47]$. The datasets were processed and analyzed using various $R$ packages for differential expression analysis. A heatmap of log2 fold change values was constructed using the heatmap $\mathrm{R}$ package (https://www.rdocumentation.org/packages/pheatmap/versions/1.0.12), and harmonized 
A

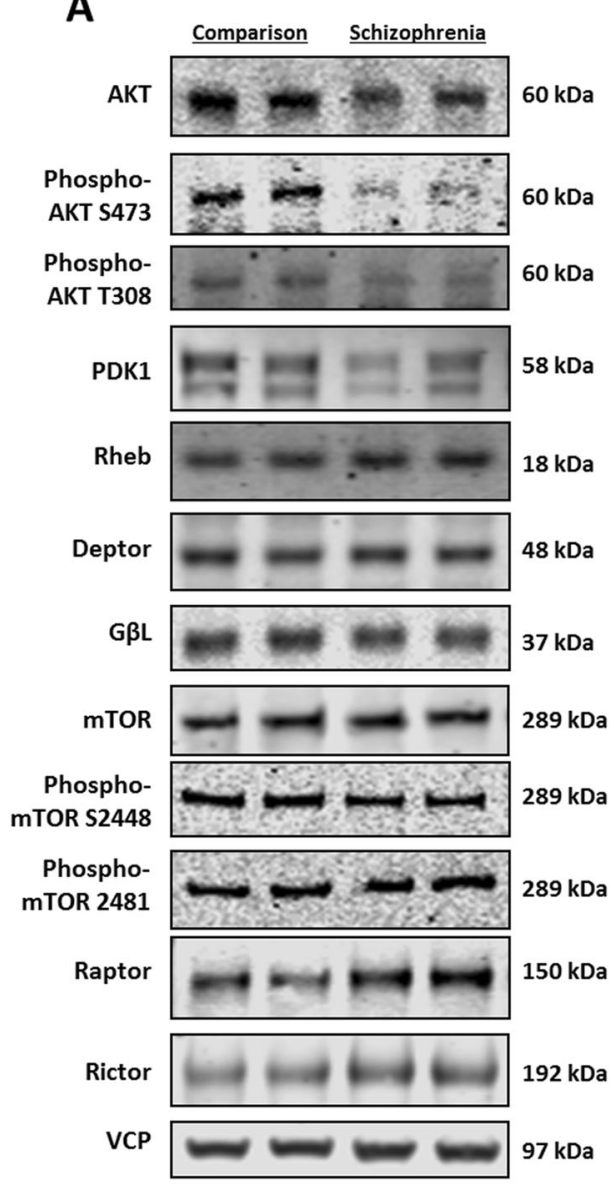

B

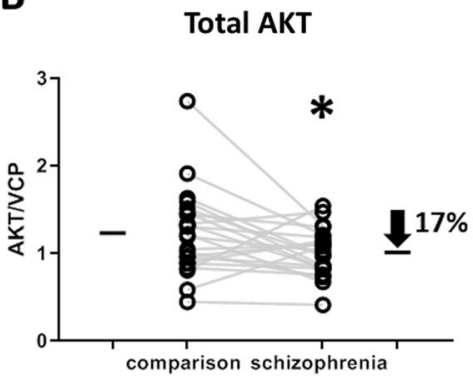

G $\beta L$

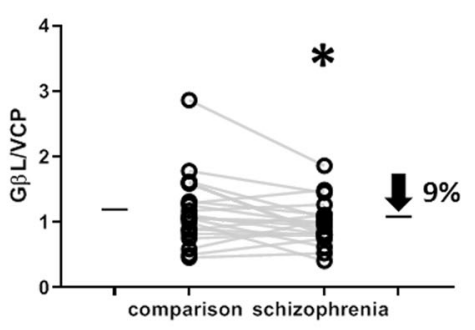

Phospho-AKT

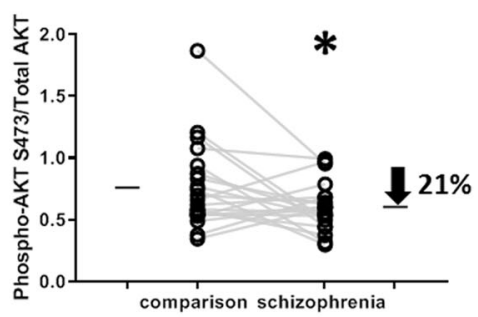

Phospho-mTOR

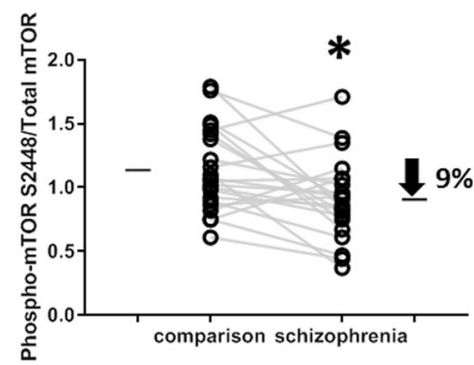

C

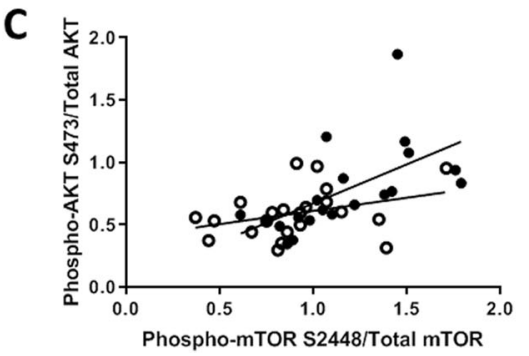

- comparison, $r=0.8^{*}$

- schizophrenia, $r=0.3$

Fig. 2 AKT-mTOR signaling pathway proteins are downregulated in SZ DLPFC. a Representative western blot analyses of protein expression of key proteins in this signaling pathway which were measured in 22 paired Comparison and Schizophrenia subjects. Phosphorylation status of AKT and mTOR as a proxy for their activity was assessed in the same subjects. $\mathbf{b}$ Decreased protein expression of AKT and G $\beta L$ was observed, whereas no change was found for expression of PDK1, Rheb, Deptor, mTOR, Raptor, or Rictor. Decreased phosphorylation of AKT at S473 and mTOR at S2448 normalized to their total protein expression was observed. c Phospho-AKT S473 was positively correlated with phospho-mTOR S2448 in comparison subjects, but not in SZ subjects. VCP was used to normalize the total expression of proteins. ${ }^{*} p \leq 0.05$.

data across different datasets were plotted to visualize the patterns of expression of key targets in the AKT-mTOR signaling pathway. Data values were harmonized using empirical cumulative probabilities based on each dataset, and final values were represented in the range of -1 to 1 in the heatmap [48].

\section{RESULTS}

Protein expression of total AKT, G $\beta \mathrm{L}$, phospho-AKT, and phosphomTOR are reduced in SZ DLPFC

We characterized the expression of the key proteins in the AKTmTOR signaling pathway in the DLPFC in paired SZ and comparison subjects (Fig. 2a). Total AKT expression $(t(21)=2.21$, $p=0.04$ ) was reduced in SZ DLPFC (Fig. 2b). We found no changes in the protein expression of PDK1, Rheb, and mTOR (Table 2). In addition, we examined the total expression of mTOR complex subunits (Table 1), and found the expression of $G \beta L(t(20)=2.38$, $p=0.03$ ) decreased in SZ (Fig. 2b). We were unable to detect and quantify PRAS40, Sin1, and Protor expression. We assessed the phosphorylation status of AKT (S473 and T308) and mTOR (S2448 and S2481) as a proxy for their activity (Fig. 2a). We observed the decreased expression of Phospho-AKT S473 normalized to total AKT expression $(t(20)=2.04, p=0.05)$ and phospho-mTOR S2448 normalized to total mTOR expression $(t(21)=2.94, p=0.01)$
(Fig. 2b). We also assessed the relationship between phosphoAKT S473 and phospho-mTOR S2448 in SZ and comparison subjects. Phospho-AKT S473 was positively correlated with phospho-mTOR S2448 in comparison subjects $(r=0.81, p<$ $0.0001)$, but not in SZ subjects ( $r=0.34, p=0.12)$ (Fig. $2 \mathrm{c}$ ).

Chronic antipsychotic treatment has no effect on protein expression and phosphorylation state in rat brain

Proteins and their phosphorylated forms found to be abnormally expressed in SZ subjects were measured in aged rats chronically treated with haloperidol decanoate. No significant changes due to haloperidol treatment were observed in the protein expression of total AKT, phospho-AKT S473, GßL, and phospho-mTOR S2448 (Fig. 3).

mTOR protein expression and phosphorylation in $\mathrm{mTORC} 1$ and mTORC2

We elucidated complex-specific abnormalities in structure and function of mTOR complexes by co-immunoprecipitation using Raptor and Rictor, key subunit proteins constituting mTORC1 and $\mathrm{mTORC2}$, respectively. The protein expression of mTOR and its phosphorylated status (S2448) was evaluated in the coimmunoprecipitated products in paired SZ and comparison subjects using Raptor/Rictor expression as the intra-lane loading control 
Table 2. Protein expression and phosphorylation status of key proteins in the AKT-mTOR signaling pathway in SZ and comparison subjects.

\begin{tabular}{|c|c|c|c|c|}
\hline Protein & Comparison & Schizophrenia & Test statistic & $p$ value \\
\hline Phospho-AKT S473/AKT & $0.76 \pm 0.08$ & $0.60 \pm 0.04$ & $t(20)=2.04$ & $0.05^{*}$ \\
\hline PDK1 & $2.13 \pm 0.29$ & $2.10 \pm 0.24$ & $t(21)=0.14$ & 0.89 \\
\hline Rheb & $1.15 \pm 0.15$ & $1.27 \pm 0.16$ & $t(20)=1.41$ & 0.17 \\
\hline Phospho-mTOR S2481/mTOR & $0.98 \pm 0.11$ & $1.04 \pm 0.18$ & $W=-25$ & 0.68 \\
\hline $\mathrm{G} \beta \mathrm{L}$ & $1.18 \pm 0.11$ & $0.98 \pm 0.07$ & $t(20)=2.38$ & $0.03^{*}$ \\
\hline Deptor & $0.07 \pm 0.01$ & $0.08 \pm 0.02$ & $W=24$ & 0.71 \\
\hline Raptor & $1.74 \pm 0.09$ & $1.74 \pm 0.15$ & $t(21)=0.05$ & 0.96 \\
\hline Rictor & $0.39 \pm 0.07$ & $0.35 \pm 0.05$ & $t(21)=0.89$ & 0.38 \\
\hline
\end{tabular}

Signal intensities of each measure from immunoblotting represented as means \pm SEM

${ }^{*} p \leq 0.05$
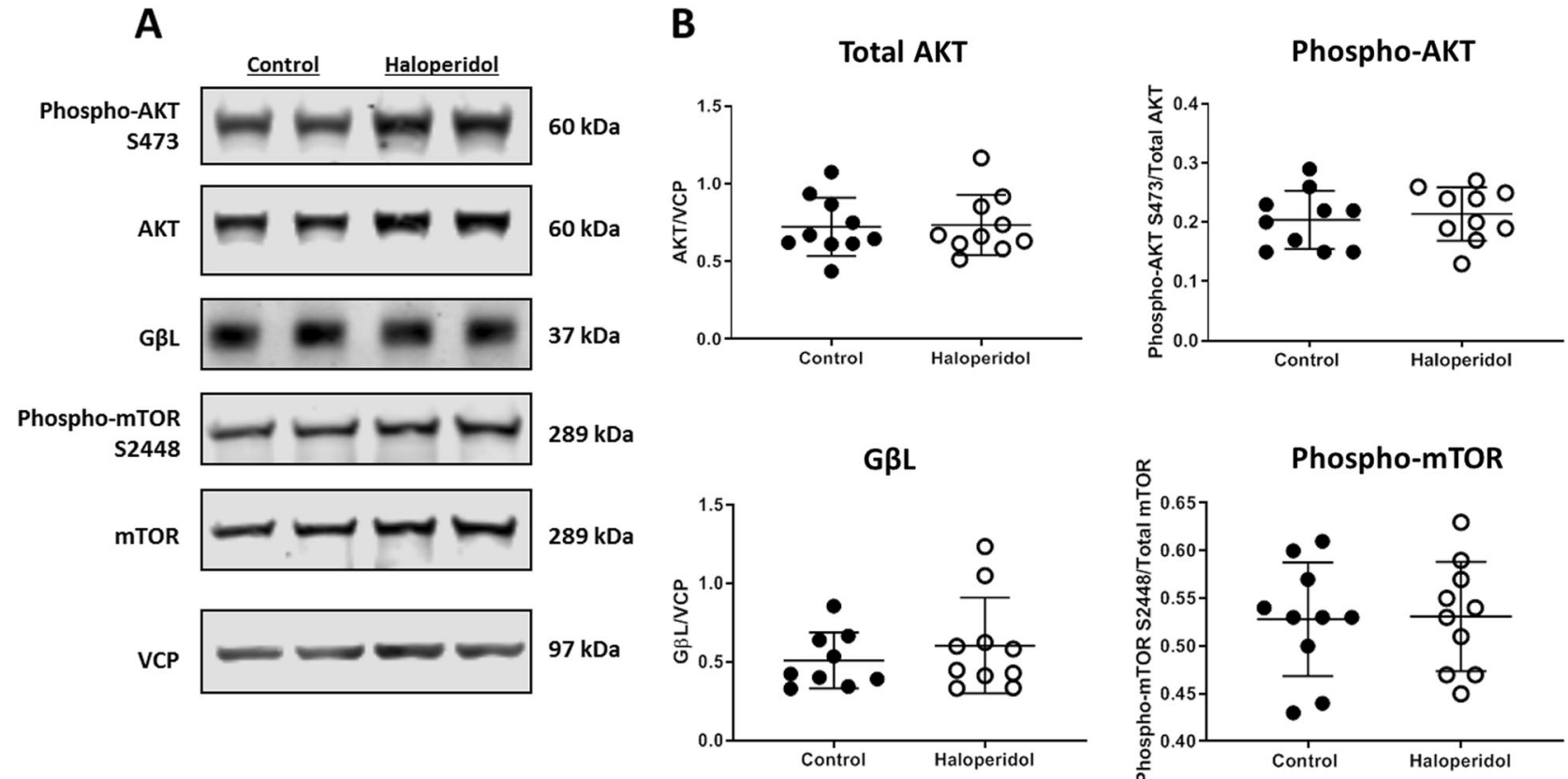

Fig. 3 No effect of chronic antipsychotic treatment on protein expression and phosphorylation in aged rats. Protein expression and phosphorylation status of targets reduced in schizophrenia were measured in rats chronically treated with haloperidol and vehicle (control). a Representative western blots from rat frontal cortex. b No changes were observed in rat frontal cortex following haloperidol treatment in expression of total AKT, phospho-AKT S473, G $\beta$ L, and phospho-mTOR. Data are shown as means \pm SEM.

(Fig. 4). We found an increased interaction of $m$ TOR in both mTORC1 $(t(20)=2.10, p=0.04)$ (Fig. $4 b)$ and mTORC2 $(t(16)=2.46$, $p=0.02$ ) (Fig. 4e). We found no change in the ratio of phosphomTOR S2448 and mTOR protein expression for either mTORC1 (Fig. 4c) and mTORC2 (Fig. 4f). We accounted for variation in coimmunoprecipitation of each complex between SZ and comparison groups by assessing the expression of the pulldown protein, Raptor or Rictor normalized to their total expression in the homogenate, and found no significant differences between diagnostic groups (Fig. S2 (B, D)).

In silico analysis

Transcript and/or protein expression of genes associated with the AKT-mTOR signaling pathway across different cell types in SZ demonstrate differential patterns of expression (Fig. 5 and Supplementary Fig. S3). In silico analysis revealed that AKT1 mRNA expression is upregulated in peripheral blood mononuclear cells obtained from SZ patients as compared with controls. PRKCA (PKC a), a downstream target of mTOR signaling, was increased at both mRNA and protein levels. Deptor and RPS6 (ribosomal protein S6) mRNA expression was decreased in iPSCs derived from SZ cases with a DISC1 mutation. Deptor is associated with mTOR in both complexes, whereas RPS6 is a downstream target of mTOR signaling. In silico analysis reveals subtle differences in expression of other genes associated with AKT-mTOR signaling in one or more of the datasets evaluated (Fig. 5).

\section{DISCUSSION}

In this study, we characterized the expression and phosphorylation status of proteins associated with the AKT-mTOR signaling pathway in DLPFC in paired SZ and comparison subjects. 


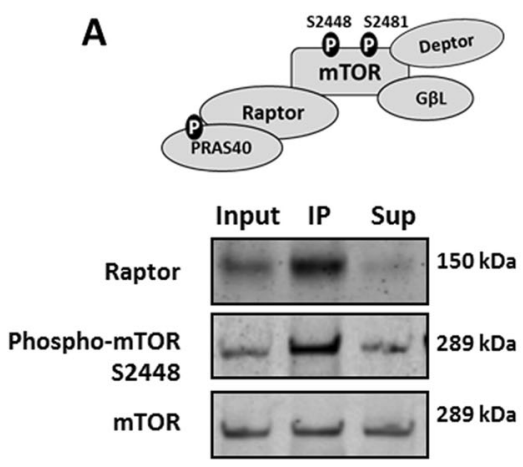

D
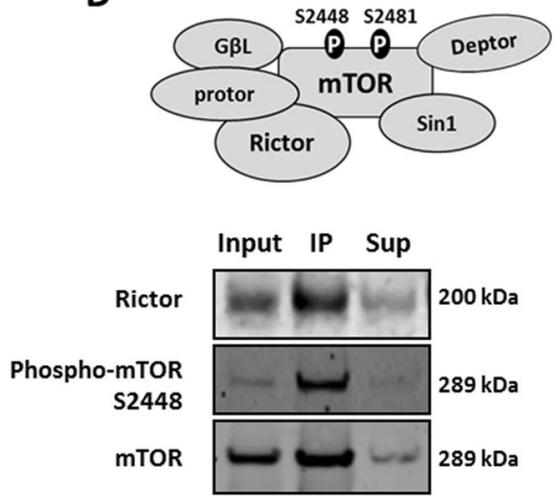

B

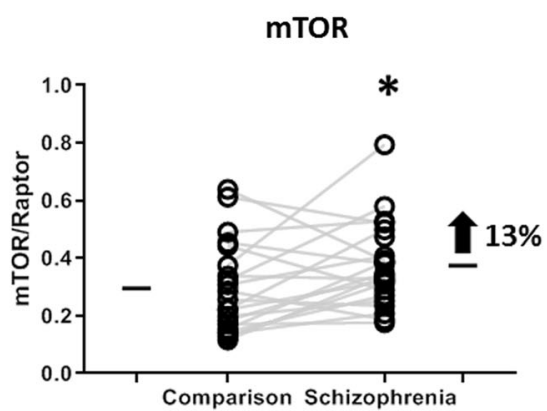

$\mathrm{E}$

mTOR

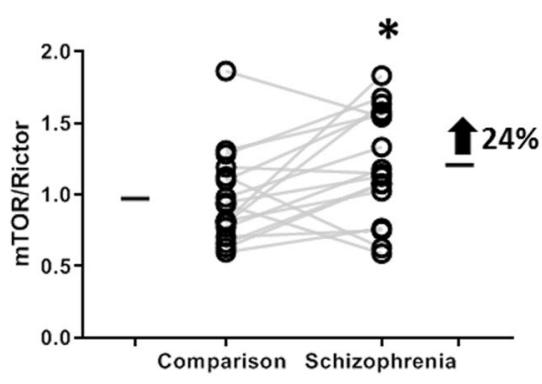

C

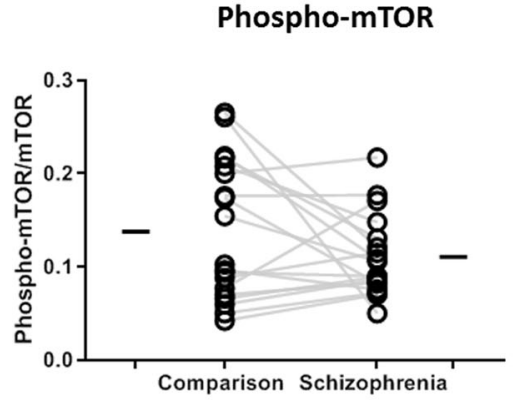

$\mathbf{F}$

Phospho-mTOR

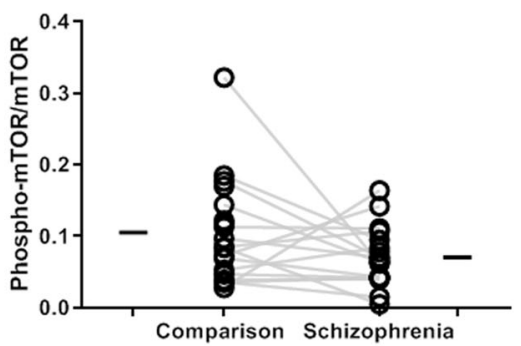

Fig. 4 mTOR protein expression and phosphorylation in mTORC1 and mTORC2. mTOR complexes were co-immunoprecipitated from comparison and schizophrenia DLPFC. a mTORC1 was co-immunoprecipitated using Raptor, and b mTOR expression and c phosphorylation status (S2448) was assessed by western blot analysis. d mTORC2 was co-immunoprecipitated using Rictor, and similarly, e mTOR expression, and $\mathbf{f}$ phosphorylation status was evaluated. We observed increased mTOR expression in both mTORC1 and mTORC2 in schizophrenia. ${ }^{*} p \leq$ 0.05 .

We identified abnormalities in the protein expression and/or phosphorylation status of AKT and mTOR kinases. AKT requires phosphorylation at both $\mathrm{S} 473$ and T308 for complete activation [15]. We observed decreased phospho-AKT S473 expression (Fig. 2b). We also observed a trend $(p=0.07)$ consistent with decreased phospho-AKT T308 expression (Table 2). AKT has been implicated in SZ pathophysiology and our results are consistent with prior reports of altered AKT expression and phosphorylation in SZ brain [29, 30, 49]. We also found that while total mTOR expression is unchanged, phosphorylation status of mTOR at S2448 is decreased in SZ DLPFC (Fig. 2b). This suggests a reduction in mTOR activity in $\mathrm{SZ}$, since it requires phosphorylation at both S2448 and S2481 for complete activation. To our knowledge, there are no prior reports of assessment of mTOR expression and phosphorylation in postmortem brain. We have utilized phosphorylation status as a proxy for kinase activity of AKT and mTOR in this study, consistent with other studies $[9,29]$. Since we observed a reduction in the phosphorylation status of both AKT and mTOR kinases, we propose that AKT-mTOR signaling is downregulated in SZ brain which may lead to functional consequences. While AKT positively regulates $\mathrm{mTORC} 1$ activity via Rheb, $\mathrm{mTORC2}$ regulates AKT activation by phosphorylation at S473 [12]. Interestingly, phospho-AKT S473 and phospho-mTOR S2448 have a strong positive correlation in nonpsychiatrically ill but not in SZ subjects (Fig. 2c). This suggests that the association between the activities of the two kinases is disrupted in SZ.

mTOR forms two different complexes that exhibit distinct functions which are dependent upon the association of specific proteins [20]. Dysregulation in the association of different proteins in these complexes likely affect their activity. Since we observed decreased expression of phospho-mTOR S2448, a modification common to the activity of both mTORC1 and MTORC2, we predicted functional consequences associated with both complexes. We also observed a reduction in the protein expression of GßL (Fig. 2b), which is incorporated into both complexes and additionally supports our hypothesis of deficits in mTOR complexes. We investigated mTOR complexes by coimmunoprecipitating them with Raptor and Rictor, and examined complex-specific subunit composition and phosphorylation in the same subjects. We found increased mTOR expression in both mTORC1 and mTORC2 (Fig. 4). Interestingly, we did not observe alterations in the expression of phospho-mTOR S2448 in either complex. This suggests that there is an increase in the recruitment of mTOR proteins into the complexes. mTOR may be differentially incorporated into the complexes and contribute to the dysregulation of complex-specific mTOR activity in SZ. Since total mTOR phosphorylation, but not complex-specific phosphorylation, was found to be changed, the increase in mTOR association with the complexes maybe due to a compensatory mechanism to restore normal functioning of downstream cellular processes in SZ subjects.

Genetic findings implicate the AKT-mTOR signaling pathway in SZ and other neuropsychiatric disorders [50]. Specifically, an AKT1 haplotype associated with lower AKT transcript and/or protein expression, is preferentially transmitted to individuals with SZ of European [29] and Japanese origin [51]. Another AKT1 haplotype has been shown to be associated with the risk of SZ in Iranian populations [52]. AKT mRNA, protein, and activity have been found to be decreased in both blood and postmortem frontal cortex in SZ [29, 30]. AKT-mTOR signaling is associated with learning and memory as well as key cellular processes that are altered in SZ. Synaptic plasticity and memory formation require new protein synthesis as well as actin dynamics-dependent changes, both of which are modulated by mTOR signaling [20]. 


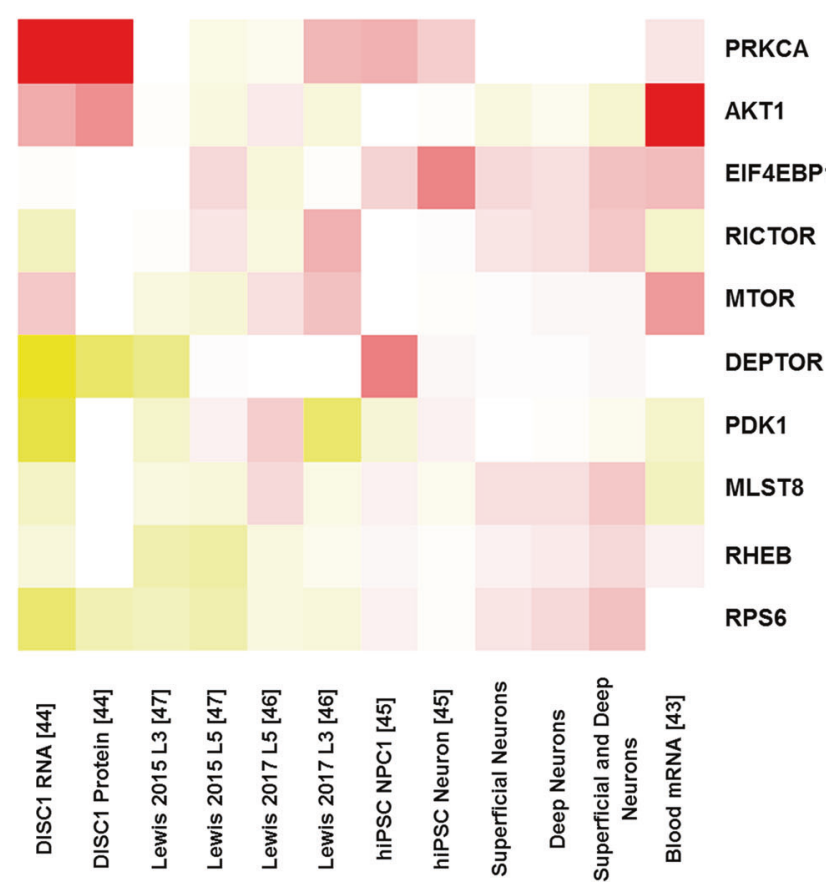

Fig. 5 Differential expression of genes associated with the AKT-mTOR signaling pathway across different cell types in SZ brain. Heatmap of log2 fold change values harmonized across different datasets. Fold change, log2 fold change, and $p$ values are reported in Supplementary Table S3. Genes with increased expression in SZ relative to comparison subjects are represented in shades of red, while genes with decreased expression are represented in shades of yellow. White represents expression not assessed for that gene in the corresponding dataset. This information was acquired from the web application Kaleidoscope, which compiles data from transcriptomic/proteomic datasets generated from different SZ models. Genes are listed on the $y$-axis and datasets are listed on the $x$-axis.

AKT knockout in mice results in deficits in hippocampal/prefrontal cortex dependent learning and synaptic plasticity [53]. Rapamycininduced inhibition of mTOR activity leads to reduced hippocampal dendritic protein synthesis, induced by the activation of glutamate receptors in rat hippocampal [54] and mouse cortical neurons [55]. Actin polymerization, assessed by measuring the ratio of filamentous (F) actin to globular (G) actin, is downregulated in mTORC2-deficient neurons, and when actin polymerization is restored in mTORC2-deficient mice, deficits in synaptic plasticity are rescued [56]. We recently demonstrated that actin polymerization is reduced in the anterior cingulate cortex in SZ [28]. Since we observed the downregulation of the AKT-mTOR signaling pathway in SZ brain, we predict that it may contribute to deficits in downstream events of protein synthesis and cytoskeletal rearrangement. Therefore, the abnormalities of protein expression, phosphorylation, and mTOR complex formation in AKT-mTOR signaling found in this study may contribute to learning and memory deficits associated with SZ. In addition, the AKT-mTOR signaling pathway is influenced by signals from neurotransmitter receptors, growth factors, and cellular stress [57]. Specifically, mTOR integrates signaling from $\mathrm{N}$-methyl-D-aspartate receptors, brain-derived neurotrophic factor and dopaminergic receptors via phosphoinositide-3 kinase, which have all been implicated in SZ $[22,58-60]$. Altogether these findings suggest that disrupted AKTmTOR signaling may lead to reduced protein synthesis and abnormal neuronal morphology, and result in a decreased brain network connectivity in SZ [61].

There are several limitations to consider in interpreting results from this study. Age, sex, $\mathrm{pH}, \mathrm{PMI}$, and chronic treatment with antipsychotic drugs are potential confounding factors which may have effects on protein expression and phosphorylation. We pairwise matched SZ and comparison subjects by sex, age, tissue $\mathrm{pH}$, and $\mathrm{PMI}$ to control for this. In addition, we performed correlation analyses to assess associations between protein expression/phosphorylation and age, $\mathrm{pH}$, and $\mathrm{PMI}$, and found no significant relationships. Some studies suggest that antipsychotic drugs may target AKT signaling. In vivo studies have suggested that acute treatment with haloperidol in rats activates GSK3ß-AKT signaling $[12,62,63]$. To control for antipsychotic treatment, we have demonstrated that chronic haloperidol treatment has no effect on protein expression and/or phosphorylation of AKT, mTOR, and G $\beta$ L in rat brain (Fig. 3). A potential limitation of the coimmunoprecipitation strategy utilized in this study is the feasibility of pulling down large mTOR complexes ( 1 MDa) $[64,65]$. mTOR complexes have been co-immunoprecipitated in cell culture studies and there were no prior reports of utilizing human postmortem brain tissue $[17,66]$. We optimized the technique using mild homogenizing conditions to maximize the likelihood that complexes remain intact and were able to demonstrate maximum depletion from the supernatant of Raptor/Rictor (Figs. 4a, d). However, due to the limitation of using frozen postmortem brain tissue, we were unable to show complete depletion of Raptor/Rictor from the homogenate and therefore, our results account for a fraction of the complexes. We also accounted for the variation in co-immunoprecipitation of Raptor and Rictor between SZ and comparison subjects and did not observe a significant difference (Supplementary Fig. S2).

An additional limitation of this study is that we measured region level but not cell-type specific protein expression. To begin to address this, we performed in silico analyses and evaluated the expression of genes associated with the AKT-mTOR signaling pathway from multiple cell-type specific datasets which are publicly available (Fig. 5 and Supplementary Table S3). We noted changes in expression in neurons in one or more datasets for some of the proteins measured in this study, including AKT. However, there is a lack of studies investigating abnormalities in AKT-mTOR signaling in nonneuronal brain cell types in SZ and related models. In addition, we cannot determine posttranslational modifications such as phosphorylation using RNA sequencing and microarray data, thus it will be critical to study signaling at the cellular level to 
develop novel and specific pharmacological targets. As evident from this in silico analysis, there are few studies in SZ research which investigate abnormalities in a cell-type specific manner. Moreover, several studies suggest that there is limited correlation between mRNA and protein expression [67, 68]. Future studies investigating the protein expression of AKT and mTOR kinases in cell-type specific populations using SZ postmortem brain and other models will be important.

In summary, we have shown the decreased expression and/or phosphorylation status of both AKT and mTOR, which suggests that the AKT-mTOR signaling pathway is downregulated in SZ brain. We have demonstrated that there are complex-specific abnormalities in mTOR expression, and mTOR complex formation and activity may be disrupted in SZ. Our findings support a model of deficits in the AKT-mTOR signaling pathway in SZ brain. Future studies to investigate the functional consequences of altered AKT-mTOR signaling cascade on intracellular processes such as protein synthesis, cytoskeleton rearrangement, and autophagy will be required to further elucidate its role in SZ.

\section{FUNDING AND DISCLOSURE}

The authors have no conflict of interest to declare. RC was supported by the Marie and Emmett Carmichael fund for graduate students in biosciences.

\section{ACKNOWLEDGEMENTS}

The authors would like to thank Dr Rosalinda Roberts and the Alabama Brain Collection for postmortem cortical samples used in assay development for western blot analyses and co-immunoprecipitation.

\section{AUTHOR CONTRIBUTIONS}

RC and JMW and designed the study. RC executed experimental protocols, performed data calculations, statistical analyses, and literature searches. RC wrote the first draft of the manuscript, followed by editing by JMW. All authors contributed to and have approved the final manuscript.

\section{ADDITIONAL INFORMATION}

Supplementary Information accompanies this paper at (https://doi.org/10.1038/ s41386-020-0614-2).

Publisher's note Springer Nature remains neutral with regard to jurisdictional claims in published maps and institutional affiliations.

\section{REFERENCES}

1. Owen MJ, Sawa A, Mortensen PB. Schizophrenia. Lancet. 2016;388:86-97.

2. Lang UE, Puls I, Müller DJ, Strutz-Seebohm N, Gallinat J. Molecular mechanisms of schizophrenia. Cell Physiol Biochem. 2007;20:687-702.

3. Meador-Woodruff JH. Novel D2-like dopamine receptors in schizophrenic brain. In: Search for the causes of schizophrenia. Heidelberg: Steinkopff; 1999. p. 251-60.

4. Joyce J, Meador-Woodruff JH. Linking the family of D2 receptors to neuronal circuits in human brain: insights into schizophrenia. Neuropsychopharmacology. 1997; 16:375-84.

5. Gao W. Dopaminergic and glutamatergic dysfunctions in the neuropathophysiology of schizophrenia. In Kudo E, Fujii Y (eds), Dopamine: functions, regulation and health effects. New York: Nova Science Publishers; 2011. p. 167-194.

6. McCullumsmith RE, Hammond J, Funk A, Meador-Woodruff JH. Recent advances in targeting the ionotropic glutamate receptors in treating schizophrenia. Curr Pharm Biotechnol. 2012;13:1535-42.

7. Rubio MD, Drummond JB, Meador-Woodruff JH. Glutamate receptor abnormalities in schizophrenia: implications for innovative treatments. Biomol Ther. 2012;20:1-18.

8. McGuire JL, Depasquale EA, Funk AJ, O'Donnovan SM, Hasselfeld K, Marwaha S, et al. Abnormalities of signal transduction networks in chronic schizophrenia. NPJ Schizophr. 2017;3:30.

9. Funk AJ, McCullumsmith RE, Haroutunian V, Meador-Woodruff JH. Abnormal activity of the MAPK- and CAMP-associated signaling pathways in frontal cortical areas in postmortem brain in schizophrenia. Neuropsychopharmacology. 2012;37:896-905.

10. Emamian ES. AKT/GSK3 signaling pathway and schizophrenia. Front Mol Neurosci. 2012;5:33.

11. Zheng W, Wang H, Zeng Z, Lin J, Little PJ, Srivastava LK, et al. The possible role of the Akt signaling pathway in schizophrenia. Brain Res. 2012;1470:145-58.

12. Kitagishi $Y$, Kobayashi M, Kikuta K, Matsuda S. Roles of PI3K/AKT/GSK3/mTOR pathway in cell signaling of mental illnesses. Depress Res Treat. 2012;2012:752563.

13. Levenga J, Wong $H$, Milstead RA, Keller BN, LaPlante LE, Hoeffer CA. AKT isoforms have distinct hippocampal expression and roles in synaptic plasticity. Elife. 2017;6. https://doi.org/10.7554/eLife.30640.

14. Radwanska K, Medvedev NI, Pereira GS, Engmann O, Thiede N, Moraes MFD, et al. Mechanism for long-term memory formation when synaptic strengthening is impaired. Proc Natl Acad Sci USA. 2011;108:18471-5.

15. Liao Y, Hung M-C. Physiological regulation of Akt activity and stability. Am J Transl Res. 2010;2:19-42.

16. Howell KR, Floyd K, Law AJ. PKBY/AKT3 loss-of-function causes learning and memory deficits and deregulation of $A K T / m T O R C 2$ signaling: Relevance for schizophrenia. PLoS ONE. 2017;12:e0175993.

17. Rosner M, Siegel AN, Valli AA, Fuchs AC, Hengstschläger AM. mTOR phosphorylated at S2448 binds to raptor and rictor. Amino Acids. 2010;38:223-8.

18. Crino PB. The mTOR signalling cascade: paving new roads to cure neurological disease. Nat Rev Neurol. 2016;12:379-92.

19. Wang $X$, Proud CG. The mTOR pathway in the control of protein synthesis. Am Physiol Soc. 2006. http://physiologyonline.physiology.org/content/nips/21/5/362. full.pdf. Accessed 21 Apr 2017.

20. Jhanwar-Uniyal M, Amin AG, Cooper JB, Das K, Schmidt MH, Murali R. Discrete signaling mechanisms of mTORC1 and mTORC2: connected yet apart in cellular and molecular aspects. Adv Biol Regul. 2017. https://doi.org/10.1016/j.jbior.2016.12.001.

21. Wiza $C$, Nascimento EBM, Ouwens DM. Role of PRAS40 in Akt and mTOR signaling in health and disease. Am J Physiol Endocrinol Metab. 2012;302:E1453-60.

22. Hoeffer CA, Klann E. mTOR signaling: at the crossroads of plasticity, memory and disease. Trends Neurosci. 2010;33:67-75.

23. Dos DS, Ali SM, Kim D-H, Guertin DA, Latek RR, Erdjument-Bromage $H$, et al. Rictor, a novel binding partner of mTOR, defines a rapamycin-insensitive and raptor-independent pathway that regulates the cytoskeleton. Curr Biol. 2004; 14:1296-302.

24. Fortin DA, Srivastava T, Soderling TR. Structural modulation of dendritic spines during synaptic plasticity. Neuroscience. 2012;18:326-41.

25. English J, Fan Y, Föcking M, Lopez L, Hryniewiecka M, Wynne K, et al. Reduced protein synthesis in schizophrenia patient-derived olfactory cells. Transl Psychiatry. 2015;5. https://doi.org/10.1038/tp.2015.119.

26. Laguesse S, Ron D. Protein translation and psychiatric disorders. Neurosci. 2019;25.

27. Glausier JR, Lewis DA. Dendritic spine pathology in schizophrenia. Neuroscience. 2013;251:90-107.

28. Bhambhvani HP, Mueller TM, Simmons MS, Meador-Woodruff JH. Actin polymerization is reduced in the anterior cingulate cortex of elderly patients with schizophrenia. Transl Psychiatry. 2017;7:1278.

29. Emamian ES, Hall D, Birnbaum MJ, Karayiorgou M, Gogos JA. Convergent evidence for impaired AKT1-GSK3 $\beta$ signaling in schizophrenia. Nat Genet. 2004;36:131-7.

30. Zhao Z, Ksiezak-Reding H, Riggio S, Haroutunian V, Pasinetti GM. Insulin receptor deficits in schizophrenia and in cellular and animal models of insulin receptor dysfunction. Schizophr Res. 2006;84:1-14.

31. Stoica L, Zhu PJ, Huang W, Zhou H, Kozma SC, Costa-Mattioli M. Selective pharmacogenetic inhibition of mammalian target of Rapamycin complex I (mTORC1) blocks long-term synaptic plasticity and memory storage. PNAS. 2011. https://doi.org/10.1073/pnas.1014715108.

32. Kim P, Scott MR, Meador-Woodruff JH. Abnormal ER quality control of neural GPIanchored proteins via dysfunction in ER export processing in the frontal cortex of elderly subjects with schizophrenia. Transl Psychiatry. 2019;9:6.

33. Scott MR, Meador-Woodruff JH. Intracellular compartment-specific proteasome dysfunction in postmortem cortex in schizophrenia subjects. Mol Psychiatry. 2019;1-15. https://doi.org/10.1038/s41380-019-0359-7.

34. Kippe JM, Mueller TM, Haroutunian V, Meador-Woodruff JH. Abnormal Nacetylglucosaminyltransferase expression in prefrontal cortex in schizophrenia. Schizophr Res. 2015;166:219-24.

35. Harte MK, Bachus SB, Reynolds GP. Increased $\mathrm{N}$-acetylaspartate in rat striatum following long-term administration of haloperidol. Schizophr Res. 2005;75:303-8.

36. Kashihara K, Sato M, Fujiwara Y, Harada T, Ogawa T, Otsuki S. Effects of intermittent and continuous haloperidol administration on the dopaminergic system in the rat brain. Biol Psychiatry. 1986;21:650-6. 
37. Hammond JC, McCullumsmith RE, Funk AJ, Haroutunian V, Meador-Woodruff JH. Evidence for abnormal forward trafficking of AMPA receptors in frontal cortex of elderly patients with schizophrenia. Neuropsychopharmacology. 2010;35:2110-9.

38. Hammond JC, Meador-Woodruff JH, Haroutunian V, McCullumsmith RE. Ampa receptor subunit expression in the endoplasmic reticulum in frontal cortex of elderly patients with schizophrenia. PLoS ONE. 2012;7:e39190.

39. Tucholski J, Simmons MS, Pinner AL, McMillan LD, Haroutunian V, MeadorWoodruff JH. N-linked glycosylation of cortical N-methyl-D-aspartate and kainate receptor subunits in schizophrenia. Neuroreport. 2013;24:688-91.

40. Pinner AL, Tucholski J, Haroutunian V, McCullumsmith RE, Meador-Woodruff JH. Decreased protein S-palmitoylation in dorsolateral prefrontal cortex in schizophrenia. Schizophr Res. 2016;177:78-87.

41. Bauer DE, Haroutunian V, McCullumsmith RE, Meador-Woodruff JH. Expression of four housekeeping proteins in elderly patients with schizophrenia. J Neural Transm. 2009;116:487-91.

42. Kim P, Scott MR, Meador-Woodruff JH. Abnormal expression of ER quality control and ER associated degradation proteins in the dorsolateral prefrontal cortex in schizophrenia. Schizophr Res. 2018;197:484-91.

43. Xu Y, Yao Shugart Y, Wang G, Cheng Z, Jin C, Zhang K, et al. Altered expression of mRNA profiles in blood of early-onset schizophrenia. Sci Rep. 2016;6. https://doi. org/10.1038/srep16767.

44. Wen Z, Nam Nguyen H, Guo Z, Lalli MA, Wang X, Su Y, et al. Synaptic dysregulation in a human iPS cell model of mental disorders HHS Public Access. Nature. 2014;515:414-8.

45. Hoffman GE, Hartley BJ, Flaherty E, Ladran I, Gochman P, Ruderfer DM, et al. Transcriptional signatures of schizophrenia in hiPSC-derived NPCs and neurons are concordant with post-mortem adult brains. Nat Commun. 2017;8. https://doi. org/10.1038/s41467-017-02330-5.

46. Arion D, Huo Z, Enwright JF, Corradi JP, Tseng G, Lewis DA. Transcriptome alterations in prefrontal pyramidal cells distinguish schizophrenia from bipolar and major depressive disorders. Biol Psychiatry. 2017;82:594-600.

47. Arion D, Corradi JP, Tang S, Datta D, Boothe F, He A, et al. Distinctive transcriptome alterations of prefrontal pyramidal neurons in schizophrenia and schizoaffective disorder. Mol Psychiatry. 2015;20:1397-405.

48. Rouillard AD, Gundersen GW, Fernandez NF, Wang Z, Monteiro CD, McDermot $M G$, et al. The harmonizome: a collection of processed datasets gathered to serve and mine knowledge about genes and proteins. Database. 2016;2016: baw100.

49. McGuire JL, Hammond JH, Yates SD, Chen D, Haroutunian V, Meador-Woodruff $\mathrm{JH}$, et al. Altered serine/threonine kinase activity in schizophrenia. Brain Res. 2014;1568:42-54.

50. Costa-Mattioli M, Monteggia LM. mTOR complexes in neurodevelopmental and neuropsychiatric disorders. Nat Publ Gr. 2013;16. https://doi.org/10.1038/nn.3546.

51. Ikeda M, Iwata N, Suzuki T, Kitajima T, Yamanouchi Y, Kinoshita Y, et al. Association of AKT1 with schizophrenia confirmed in a Japanese population. Biol Psychiatry. 2004;56:698-700.

52. Bajestan SN, Sabouri AH, Nakamura M, Takashima H, Keikhaee MR, Behdani F, et al. Association of AKT1 haplotype with the risk of schizophrenia in Iranian population. Am J Med Genet Part B Neuropsychiatr Genet. 2006;141B:383-6.
53. Balu DT, Carlson GC, Talbot K, Kazi H, Hill-Smith TE, Easton RM, et al. Akt1 deficiency in schizophrenia and impairment of hippocampal plasticity and function. Hippocampus. 2012;22:230-40.

54. Gong R, Park CS, Abbassi NR, Tang S-J. Roles of glutamate receptors and the mammalian target of rapamycin (mTOR) signaling pathway in activity-dependent dendritic protein synthesis in hippocampal neurons. J Biol Chem. 2006;281:18802-15

55. Hsu W-L, Chung H-W, Wu C-Y, Wu H-I, Lee Y-T, Chen E-C, et al. Glutamate stimulates local protein synthesis in the axons of rat cortical neurons by activating a-amino-3-hydroxy-5-methyl-4-isoxazolepropionic acid (AMPA) receptors and metabotropic glutamate receptors. J Biol Chem. 2015;290:20748-60.

56. Huang W, Zhu PJ, Zhang S, Zhou H, Stoica L, Galiano M, et al. mTORC2 controls actin polymerization required for consolidation of long-term memory. Nat Neurosci. 2013;16:441-8.

57. Wang L, Zhou K, Fu Z, Yu D, Huang H, Zang X, et al. Brain development and Akt signaling: the crossroads of signaling pathway and neurodevelopmental diseases. J Mol Neurosci. 2017;61:379-84.

58. Kristiansen LV, Beneyto M, Haroutunian V, Meador-Woodruff JH. Changes in NMDA receptor subunits and interacting PSD proteins in dorsolateral prefrontal and anterior cingulate cortex indicate abnormal regional expression in schizophrenia. Mol Psychiatry. 2006;11:737-47.

59. Thomas KT, Anderson BR, Shah N, Zimmer SE, Hawkins D, Valdez AN, et al Inhibition of the schizophrenia-associated microRNA miR-137 disrupts Nrg1a neurodevelopmental signal transduction. Cell Rep. 2017;20:1-12.

60. Funk AJ, Rumbaugh G, Harotunian V, McCullumsmith RE, Meador-Woodruff JH. Decreased expression of NMDA receptor-associated proteins in frontal cortex of elderly patients with schizophrenia. Neuroreport. 2009;20:1019-22.

61. Gururajan A, Van Den Buuse M. Is the mTOR-signalling cascade disrupted in Schizophrenia? J Neurochem. 2014;129:377-87.

62. Roh M-S, Seo MS, Kim Y, Kim SH, Jeon WJ, Ahn YM, et al. Haloperidol and clozapine differentially regulate signals upstream of glycogen synthase kinase 3 in the rat frontal cortex. Exp Mol Med. 2007;39:353-60.

63. Pan B, Huang XF, Deng C. Aripiprazole and haloperidol activate GSK3 3 dependent signalling pathway differentially in various brain regions of rats. Int J Mol Sci. 2016;17. https://doi.org/10.3390/ijms17040459.

64. Sarbassov DD, Bulgakova O, Bersimbaev RI, Shaiken T. Isolation of the mTOR complexes by affinity purification. In: Methods in molecular biology. Humana Press; 2012. p. 59-74.

65. Jain A, Arauz E, Aggarwal V, Ikon N, Chen J, Ha T. Stoichiometry and assembly of mTOR complexes revealed by single-molecule pulldown. Proc Natl Acad Sci USA. 2014;111:17833-8.

66. Dos DS, Ali SM, Kim D-H, Guertin DA, Latek RR, Erdjument-Bromage $\mathrm{H}$, et al. Rictor, a novel binding partner of $\mathrm{mTOR}$, defines a rapamycin-insensitive and raptorindependent pathway that regulates the cytoskeleton. Curr Biol. 2004;14:1296-302.

67. De Sousa Abreu R, Penalva LO, Marcotte EM, Vogel C. Global signatures of protein and mRNA expression levels. Mol Biosyst. 2009;5:1512-26.

68. Macdonald ML, Garver M, Newman J, Sun Z, Kannarkat J, Salisbury R, et al. Synaptic proteome alterations in the primary auditory cortex of individuals with schizophrenia. JAMA Psychiatry. 2019. https://doi.org/10.1001/ jamapsychiatry.2019.2974. 